\title{
Chronic Abdominal Pain in Children and Adolescents: Parental Threat Perception Plays a Major Role in Seeking Medical Consultations
}

\author{
Claudia Calvano and Petra Warschburger \\ Department Psychology, Counselling Psychology, University of Potsdam, Potsdam, Germany \\ Correspondence should be addressed to Claudia Calvano; calvano@uni-potsdam.de
}

Received 16 March 2016; Revised 19 October 2016; Accepted 8 November 2016

Academic Editor: Jacob Ablin

Copyright (c) 2016 C. Calvano and P. Warschburger. This is an open access article distributed under the Creative Commons Attribution License, which permits unrestricted use, distribution, and reproduction in any medium, provided the original work is properly cited.

Background. Pain symptoms, associated impairment, and parental perception of threat are reported to be predictors of health care utilization (HCU) in childhood chronic abdominal pain (CAP). However, mediating variables and their interrelations have not yet been systematically studied. Objectives. This study aims to identify mediating pathways of influence between child's abdominal pain and the number of pain-related medical visits. Methods. In a multicenter study, we recruited $N=151$ parent-child dyads with children aged 6-17 years suffering from CAP. A composite measure of pain symptoms was defined as predictor and the number of pain-related medical visits as outcome variable. This relation was analyzed by serial mediation, including child- and parent-reported impairment and parental threat perception as mediators. Results. Only parental threat perception significantly linked child's pain symptoms to the number of medical visits. Measures of impairment did not have a significant effect. Conclusions. Parental painrelated threat perception is strongly related to health care seeking in childhood CAP. Addressing threat perception might be a fruitful parent-centered approach in clinical practice.

\section{Introduction}

Chronic abdominal pain (CAP) is one of the most frequent bodily complaints in childhood and adolescence $[1,2]$ and is associated with a high psychosocial burden, poor functioning, and low health-related quality of life [3, 4]. Furthermore, CAP represents the most common reason for consultations with pediatric gastroenterologists [5], and children with the condition show higher health care utilization than children suffering from headaches or other bodily symptoms [6]. Increased utilization of health care not only poses an economic burden due to repeated diagnostic procedures [7] and referral to higher-level medical services [8] but may also negatively influence the prognosis of child pain $[9,10]$.

Therefore, research in this area has concentrated on identifying predictors of pediatric health care utilization (HCU). Both the level of pain symptoms and the degree of painrelated impairment in everyday functioning are positively correlated with the number of pediatric visits $[2,11-16]$.
Studies taking both of these variables into account often reported a greater impact from the level of impairment than from pain per se $[2,17]$. However, the assessment of pain-related impairment varied in terms of the source of information, mostly depending on the age of the sample. Many studies including a broad age range were unable to differentiate between child- and parent-reported impairment, as assessment depended on the child's age, with parent report used for younger children and self-report used for children aged 11 or older [14]. Moreover, in adolescent samples, some studies included only self-report (e.g., [17]) while others relied solely on parent report [18]. According to results by Toliver-Sokol et al. [15] from an adolescent chronic pain sample, only the parent report on activity restrictions, and not the adolescent self-report, was directly related to HCU. Therefore, the first research question of this study was to analyze whose perception of pain-related impairment in everyday life, the one of the child or the one of the parent, might better explain health care seeking. 
The second research question concerns the influence of parental beliefs regarding child's abdominal pain on health care seeking. Parental factors play an important role for HCU in childhood pain which was highlighted by several studies: Levy and colleagues [13] identified maternal psychological distress, that is, increased scores for anxiety, depression, or somatization, as strongest predictor for medical consultations, beyond child-reported pain. Taking also into account the study by Venepalli and colleagues [16], one can conclude that not only mother's own psychological strain [13] but also that maternal fear specifically regarding child's pain and longterm impairment influences HCU [16]. These beliefs relating to the significance of child's chronic pain are captured by the construct of parental threat perception regarding child's pain.

Evidence regarding parental threat perception in childhood chronic pain can be derived from various research contexts, that is, experimental, observational, and interventional studies. For instance, in experimental research, parental threat perception was induced by delivering threatening information regarding child's pain in the trials [19-21]. When parents are to believed that the pain imposes a high threat on the child, then not only did the parents expect higher pain levels for the child [19], but also parents were more attentive to the child $[20,21]$. In studies assessing parental threat perception by questionnaire, it has been observed that increased levels of parental threat perception correspond with increased child pain intensity and a poorer adaptation profile [22]. Furthermore, Levy and colleagues showed that parental threat perception was significantly reduced after participation in a cognitive-behavioral intervention for functional abdominal pain $[23,24]$. Due to variations in study design, the operationalization of threat perception differs across studies. Questionnaire assessment mostly operationalized threat perception as composite score of perceived pain symptom level (like intensity, duration, and frequency) and also the perceived seriousness and long-term impact of pain for child's health [22-24]. Regarding the latter dimension on pain as threat for child's long-term health, the context of health psychology research gives further evidence. In that field, threat perception is defined by two components: perceived vulnerability (or susceptibility) to illness and perceived severity (or seriousness) of symptoms [25]. This definition of threat perception has emerged as one key factor for the initiation of health-related behaviors [26]. Accordingly, parental threat perception seems to be a very promising construct to explain HCU in childhood pain as well. Up to now, in pediatric pain research, there is evidence that appraisals of the child's health status $[11,12]$ and child vulnerability for illness [27] significantly contribute to care seeking as well. However, the role of parental threat perception in childhood CAP for health care utilization has not been analyzed so far.

To date, studies have mainly examined direct effects on HCU, thus precluding statements about possible mediating variables. Moreover, most studies included either the child's or parents' view on impairment, thus impeding evidence on whose perception of impairment might be more relevant for HCU. To extend current knowledge on child and parent influences on HCU, this study

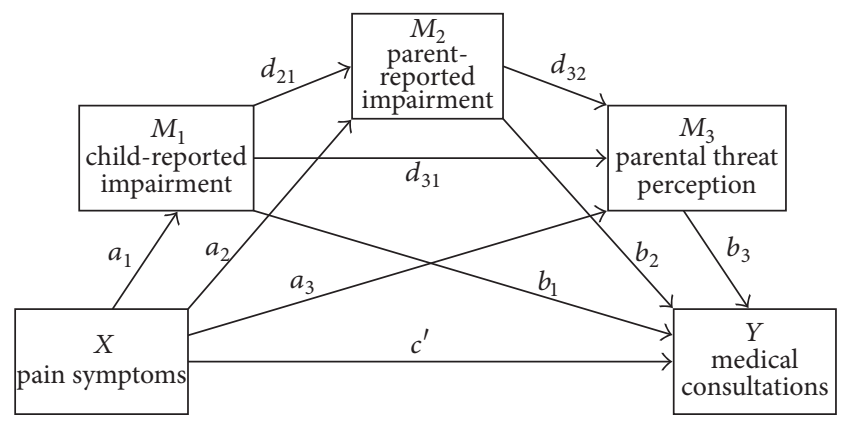

FIgURE 1: Conceptual model for serial mediation in the relation between child's pain symptoms and medical consultations, including coefficients for the direct pathways ( $a_{i}$ for pathways between predictor pain symptoms and each mediator $M_{i} ; b_{i}$ for pathways between each mediator $M_{i}$ and the outcome medical consultations; $d_{i j}$ for serial pathways between the mediators; $c^{\prime}$ for the direct effect between pain symptoms as predictor and medical consultations as outcome).

applies a concurrent analysis of child and parent mediators of HCU in a sample suffering from chronic abdominal pain.

Based on current evidence [2,13-15], we first assume a direct positive association between the child's pain symptoms and the number of pediatric visits. We aim to explain this relationship by including impairment and parental threat perception as mediators. In order to assess distinct influences, we include both child and parent reports of impairment in the model. Literature reviews on the interplay between child and parent factors in chronic pain (e.g., [28]) form the basis for our conceptual model. Assuming that parent's cognitive and behavioral reactions depend on child's pain experience and associated behavior, we postulate a serial mediation model as follows: child-reported impairment is defined as first mediating variable, explaining the link between pain symptoms and medical visits. As we hypothesize that the degree of parent's reported impairment is influenced by child's pain and impairment, this variable was defined as second mediator. Assuming that parental threat perception is based on the observation of the child's pain symptoms and influenced by the degree of impairment in everyday functioning, we postulate a serial mediation model for the interactive effects of these variables (Figure 1).

Using this model, specific effects of each mediator are analyzed separately as well as serially. We hypothesize (1) that impairment will mediate the relationship between pain reports and medical visits, (2) that when both child and parent report of impairment are taken into account, parentreported impairment will exert the stronger influence, and (3) that threat perception will significantly explain additional variance.

Furthermore, we compare the strengths of the indirect effects by contrasting the coefficients. We hypothesize that the indirect pathways including threat perception as mediator $\left(M\right.$; including $M_{3}, M_{1}-M_{3}, M_{2}-M_{3}$, and $\left.M_{1}-M_{2}-M_{3}\right)$ will exert a more pronounced influence than the pathways which only control for threat perception $\left(M_{2}, M_{1}-M_{2}\right)$. 


\section{Methods}

2.1. Design. This cross-sectional study is part of a research project focusing on childhood CAP [4]. In 16 study centers, children suffering from chronic abdominal pain and their caregivers were consecutively recruited during visits to a pediatric gastroenterological outpatient clinic. Children underwent medical examination conducted by the pediatric gastroenterologist who also reported on their gastrointestinal diagnosis. Data for the research project were collected by questionnaires after parental informed consent and child assent. Over a 9-month period, 635 families with children aged 1-18 years presenting at the clinic were addressed, of which $34.8 \%$ complete parent-child dyads were returned. For this study, the inclusion criteria were as follows: age of child 6-17 years, presentation due to chronic abdominal pain, child and parent data available, informed parental consent, and child assent.

This study was approved by the Ethics Committee of the University of Potsdam (date 18/04/2011).

2.2. Participants. $N=151$ parent-child dyads fulfilled inclusion criteria. Table 1 summarizes the sociodemographic and utilization-related characteristics of the final sample. The medical diagnoses were as follows: $7.3 \%$ lactose intolerance, $27.8 \%$ fructose malabsorption, $7.9 \%$ both, $2.0 \%$ other carbohydrate malabsorption, $5.3 \%$ constipation, and $49.7 \%$ functional gastrointestinal disorder. We assessed possible differences between diagnostic groups (carbohydrate intolerance/constipation versus functional gastrointestinal disorder) on the main variables in our mediation model. Results are summarized in Table 2 . As subgroups do not significantly differ on the measures, we combined the groups in one analysis.

\subsection{Measures}

Pain Symptoms. The children rated their pain intensity ("How strong was your belly ache during the last 2 weeks?") and pain frequency ("How often did you have belly ache during the last 2 weeks?") on a 6-point scale with verbal descriptions (from 0 "not at all" to 5 "very strong"/ "daily"); the pain duration ("How long does your belly ache usually last?") was rated on a 5 -point scale with verbal descriptions (from 1 "less than one hour" to 5 "the whole day"). For the items on intensity and frequency, we used the faces derived from Wong-Baker Faces Pain Rating Scale (WBFPRS [29]) as additional answering format corresponding to the verbal descriptions of categories. The use of the WBFPRS served to take the broad age span into account and ease answering for the younger children in the sample. In a systematic review, this scale was reported as valid pain measure, highly accepted by children and therefore recommended for children from the age of 6 onwards [30]. Ratings of intensity, frequency, and duration were multiplied to form a comprehensive index of abdominal pain symptoms (cf. [31]).

Pain-Related Impairment Child Self-Report. We used the "interference" subscale of the Pain Experience Questionnaire
TABLE 1: Sample characteristics ( $N=151$ parent-child dyads).

\begin{tabular}{|c|c|}
\hline Variable & \\
\hline \multicolumn{2}{|l|}{ Child age } \\
\hline M & 10.95 \\
\hline$(\mathrm{SD})$ & $(2.64)$ \\
\hline Range & $6-17$ \\
\hline \multicolumn{2}{|l|}{ Parent age } \\
\hline M & 41.92 \\
\hline$(\mathrm{SD})$ & $(5.91)$ \\
\hline Range & $27-56$ \\
\hline \multicolumn{2}{|l|}{ Gender child } \\
\hline$\%$ (female/male) & $64.9 / 35.1$ \\
\hline \multicolumn{2}{|l|}{ Gender parent } \\
\hline$\%$ (female/male) & $85.4 / 14.6$ \\
\hline \multicolumn{2}{|l|}{ Single parent } \\
\hline$\%$ (yes/no) & $17.2 / 82.8$ \\
\hline \multicolumn{2}{|l|}{ Education parent } \\
\hline$\%$ (low/medium/high) & $18.5 / 41.1 / 40.4$ \\
\hline \multicolumn{2}{|l|}{ Duration since onset } \\
\hline$\%(<3 / 4-11 / \geq 12$ mo. $)$ & $19.2 / 30.5 / 50.3$ \\
\hline \multicolumn{2}{|c|}{ Total duration of medical care (months) } \\
\hline $\mathrm{M}$ & 17.40 \\
\hline$(\mathrm{SD})$ & $(27.22)$ \\
\hline Range & $0-132$ \\
\hline \multicolumn{2}{|c|}{$\begin{array}{l}\text { Number of different physicians seen within } \\
\text { last } 6 \text { months }\end{array}$} \\
\hline M & 3.98 \\
\hline (SD) & $(3.36)$ \\
\hline Range & $1-25$ \\
\hline \multicolumn{2}{|l|}{ Low versus high utilizer } \\
\hline$\%$ (low/high) & $62.9 / 37.1$ \\
\hline
\end{tabular}

Note. High utilizer $\geq 4$ visits in last 6 months; education was coded as low (no school-leaving qualifications or special school), medium (secondary school), and high (diploma or university degree).

[32], a validated measure for children and adolescents aged 7-18 years. The six items cover school and homework, leisure time, and family activities. The scale score correlates with pain measures and internalizing symptoms [32]. The original 7 -point scale was changed into a 5-point Likert scale (from 1 "never" to 5 "always") in order to correspond to the 5point format of other scales in the child questionnaire [4]. We calculated a sum score with good internal consistency in our sample (Cronbach $\alpha=.89$ ).

Pain-Related Impairment Parent Report. We used the parent version of the Pediatric Pain Disability Index (P-PDI [33]), which is a validated proxy report for adolescents aged 11-17 years. Parents rated the degree of their child's impairment in everyday life (12 items; e.g., reading, going to school, and sleeping) on a 5-point frequency scale (from "never" to "always"). We calculated a sum score, which yielded excellent internal consistency in our sample (Cronbach $\alpha=.92$ ). 
TABLE 2: Descriptive data and results of group comparisons on measures included in the meditation model $(N=151)$.

\begin{tabular}{|c|c|c|c|c|c|}
\hline & \multicolumn{2}{|c|}{$\begin{array}{l}\text { Carbohydrate intolerance and constipation } \\
\text { (CC) } N=76\end{array}$} & \multicolumn{2}{|c|}{$\begin{array}{l}\text { Functional gastrointestinal disorder (FGID) } \\
\qquad N=75\end{array}$} & \multirow[t]{2}{*}{$p$} \\
\hline & $\mathrm{M}(\mathrm{SD})$ & Range & $\mathrm{M}(\mathrm{SD})$ & Range & \\
\hline Medical consultations $^{1}$ & $3.80(3.40)$ & $1-25$ & $4.15(3.33)$ & $1-20$ & 0.544 \\
\hline Pain symptoms & $29.42(30.98)$ & $0-125$ & $31.74(28.88)$ & $0-125$ & 0.636 \\
\hline Impairment self-report & $49.60(29.12)$ & $0-100$ & $56.33(26.17)$ & $0-100$ & 0.137 \\
\hline Impairment parent-report & $35.89(20.57)$ & $0-100$ & $36.14(19.13)$ & $0-79.17$ & 0.937 \\
\hline Threat perception & $47.22(23.10)$ & $0-100$ & $47.83(22.85)$ & $0-100$ & 0.871 \\
\hline
\end{tabular}

Note. Except for health care utilization and pain symptoms, scale scores were transformed to a range from 0 to $100 .{ }^{1}$ Medical consultations refer to the number of consultations due to the child's abdominal pain in the last 6 months (due to missing dataN = 68 for HCU in the CC group; $N=70$ for HCU in the FGID group).

Threat Perception. In this study, threat perception was defined as a two-component construct including perceived vulnerability for long-term illness and perceived severity of abdominal pain for child's health. Based on established measures for threat perception in adult samples [25, 34], we adapted the wording of the two items for perceived vulnerability, respectively, perceived severity to the context of childhood CAP. Parents reported on their child's vulnerability ("How high do you rate the risk of long-term impairment for your child because of abdominal pain?"; cf. [35]) and on perceived severity of pain for child's health ("How severe do you rate the abdominal pain for the health of your child?"; cf. [36]). For each item, scaling ranged from 1 to 7 , with anchors on 1 (not very likely/not severe), 4 (moderately likely/moderately severe), and 7 (very likely/very severe). Threat perception was computed as a mean score, with sufficient internal consistency (Cronbach $\alpha=.77$ ).

Health Care Utilization. In line with the literature $[13,15]$, health care utilization was assessed using an open-response format based on parent report ("In the last 6 months, how often did you visit a doctor because of your child's abdominal pain?").

Sociodemographic Factors. Children's age and gender were collected based on parent report. Parents also reported their marital status, educational background, and provided descriptive data on the child's health care use.

2.4. Data Analysis. The final dataset was analyzed using SPSS 22 for Windows. The serial mediation analysis based on OLS regression was conducted using the PROCESS macro for SPSS by Hayes [37, 38]. Missing values in latent variables were substituted with the EM algorithm when the proportion of missing data per variable did not exceed 5\% [39], which applied for the predictor and all the three mediator variables in the conceptual model. For the outcome variable of medical consultations due to abdominal pain, there were missing data in 10 cases $(6.6 \%)$, exceeding the threshold for substitution set in this study. Therefore, these missing values were not replaced by EM imputation. As the PROCESS macro does not allow for missing data, for mediation analysis and bootstrapping, these 10 cases were excluded from analysis; in addition, 3 cases had to be excluded due to indistinct reports on HCU (no visits in the last 6 months) which results in total $N=138$ for the serial mediation analysis.

Our model is based on a direct effect of pain symptoms on medical consultations $\left(c^{\prime}\right)$. According to our hypotheses, impairment based on child self-report was defined as first mediator $\left(M_{1}\right)$, followed by parent report of impairment $\left(M_{2}\right)$ and parental threat perception $\left(M_{3}\right)$, resulting in three specific indirect effects, through $M_{1}\left(a_{1} b_{1}\right), M_{2}\left(a_{2} b_{2}\right)$ or $M_{3}$ $\left(a_{3} b_{3}\right)$. In addition, effects in sequence were analyzed, that, is over the sequence $M_{1}-M_{2}\left(a_{1} d_{21} b_{2}\right), M_{1}-M_{3}\left(a_{1} d_{31} b_{3}\right)$, and $M_{2}-M_{3}\left(a_{2} d_{32} b_{3}\right)$ or over all mediators in serial: $M_{1}-M_{2}-M_{3}$ $\left(a_{1} d_{21} d_{32} b_{3}\right)$. Notably, in each subanalysis, the remaining mediator(s) were statistically controlled for. Pairwise comparisons of coefficients (contrasts) examined the strength of effects, that is, whether and how indirect effects differed from each other. Statistical significance was based on 95\% biascorrected and accelerated confidence intervals for the regression coefficient (95\% BCa CI) [40], using 10.000 bootstrap samples.

\section{Results}

Due to missing cases in the outcome "medical consultations," the sample size in statistical analyses and hypotheses testing refers to $N=138$. Bivariate correlations and descriptive statistics for the variables in the model are summarized in Table 3. In the first step, we checked correlations with main sociodemographic measures (age, gender, and socioeconomic status) to identify possible covariates in the model. The number of medical consultations was significantly correlated with the child's age $(r=0.216, p=0.011)$, but not with the parents' age $(r=0.114, p=0.184)$. As the child's age was also significantly correlated with pain symptoms $(r=0.224, p=$ $0.006)$, parent-reported impairment $(r=0.279, p<0.001)$, and threat perception $(r=0.243, p=0.003)$, we included the child's age as covariate in the analysis. There were no significant correlations with parent gender or socioeconomic status.

The results with respect to the single pathways in the model (see Figure 1) are summarized in Table 4. In line with the conceptual model, we found a significant association between the child's pain and number of medical consultations 
TABLE 3: Descriptive statistics and bivariate correlations $(N=151)$.

\begin{tabular}{|c|c|c|c|c|c|c|c|}
\hline & Variable & 2 & 3 & 4 & 5 & $\mathrm{M}(\mathrm{SD})$ & Range \\
\hline 1 & Medical consultations $^{1}$ & $0.228^{* *}$ & $0.201^{*}$ & $0.246^{* *}$ & $0.313^{* * *}$ & $3.98(3.36)$ & $1-25$ \\
\hline 2 & Pain symptoms & - & $0.302^{* * *}$ & $0.434^{* * *}$ & $0.457^{* * *}$ & $30.57(29.87)$ & $0-125$ \\
\hline 3 & Impairment self-report & - & - & $0.376^{* * *}$ & $0.256^{* *}$ & $52.94(27.81)$ & $0-100$ \\
\hline 4 & Impairment parent-report & - & - & - & $0.391^{* *}$ & $36.01(19.80)$ & $0-100$ \\
\hline 5 & Threat perception & - & & - & - & $47.53(22.90)$ & $0-100$ \\
\hline
\end{tabular}

Note. Except for health care utilization and pain symptoms, scale scores were transformed to a range from 0 to $100 .{ }^{*} p<0.05,{ }^{* *} p<0.01$, and ${ }^{* * *} p<0.000$.

${ }^{1}$ Medical consultations refer to the number of consultations due to the child's abdominal pain in the last 6 months $(N=138$ due to missing data on medical consultations).

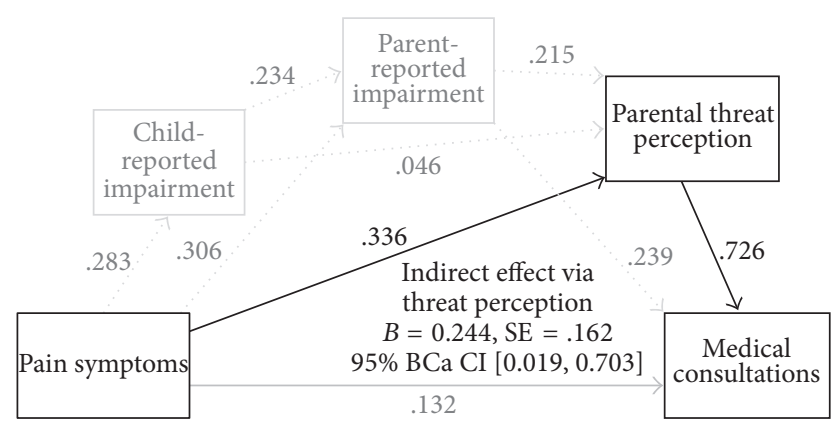

Figure 2: Final mediation model for the relation between pain symptoms and medical consultations with coefficients for the direct pathways $(N=138)$. Pathways marked in grey indicate the nonsignificant effects.

(total effect $B=0.621, \mathrm{SE}=0.287, p=0.032,95 \% \mathrm{BCa} \mathrm{CI}$ $[0.054,1.188])$, explaining $7.8 \%$ of the variance $(F(2,135)=$ $5.752, p=0.004)$. When the mediators were included in the analysis, this coefficient was no longer statistically significant (direct effect $c^{\prime} ; B=0.132, \mathrm{SE}=0.326, p=0.687,95 \% \mathrm{BCa} C \mathrm{CI}$ $[-0.513,0.776])$.

The results for the specific and serial mediation effects are summarized in Table 5. Summing up all indirect effects yielded a significant result (total indirect effect, $B=0.489$, SE $=0.226,95 \%$ BCa CI $[0.125,1.008])$. In line with hypothesis 1 , self-report of impairment did not have a significant specific indirect effect when parental measures were controlled for. In contrast to hypothesis 2 , proxy report of impairment also did not have a specific effect. Threat perception proved to be a significant mediator, which is in line with hypothesis 3 . For serial effects, no pathway yielded statistical significance. As we only had one statistically significant mediation effect, contrasts were suspended with. The final model is depicted in Figure 2.

\section{Discussion}

We analyzed influences on children's medical consultations in an outpatient, secondary care sample of children and adolescents suffering from CAP. By integrating self- and parent-reported impairment and parental threat perception simultaneously, this analysis extended the current knowledge on predictors of pediatric health care seeking in three ways.
First, the concurrent analysis of both child- and parentreported impairment provided insight into the relevance of the parents' view independent of the child's age; second, the concurrent analysis enabled conclusions to be drawn about the influence of parental threat perception beyond impairment; and third analyzing parents' threat perception provided first insight into its importance for medical utilization in childhood CAP.

In line with the literature, pain symptoms were strongly and positively correlated with the number of medical consultations $[11,14,15]$. In agreement with our hypothesis, the influence of child-reported impairment disappeared when parental measures were taken into account. This underlines that parents' view on impairment plays a major role in explaining pediatric consultations $[12,15,16]$. In addition, we found different contributions of perceived impairment and threat perception. Despite high coefficients on direct pathways in the model for both variables, only parental threat perception significantly linked pain symptoms and number of medical visits. This observation extends previous studies reporting that pain symptoms and related impairment are stable predictors of $\operatorname{HCU}[2,6,11,14,15]$ insofar as we now point to the dominant mediating role of parental threat perception for HCU.

Our study should be discussed in light of the results by Venepalli et al. [16], who identified that maternal fear of persistence and long-term impairment differentiated between consulting versus nonconsulting families. Focusing on consulting families, we have now identified threat perception, which comprises comparable worries, as the major influence for seeking medical visits. We also add to results by Connelly et al. [27], who identified parental perceptions of child vulnerability to illness as a mediating variable for HCU, by operationalizing threat perception as a very painspecific measure, taking perceptions of vulnerability and severity of the child's pain into account. The role of parent's threat perception regarding child's pain is underlined in a variety of studies. While the results for this study identified threat perception as central mediator for medical health care seeking, the observations in an interventional study by Levy and colleagues deliver further evidence as well. Applying three common parent and child sessions based on social learning theory, the authors not only report that parental threat perception was significantly reduced after treatment $[23,24]$, but also identified reductions in parental threat perception as mediator, explaining the decrease in 


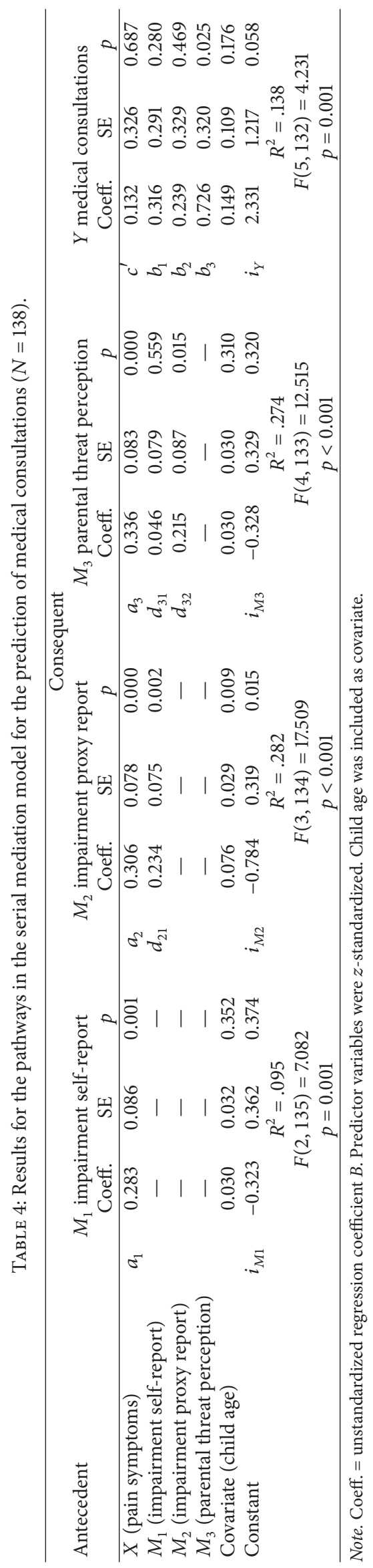


TABLE 5: Results of serial mediation analysis for specific and serial indirect effects $(N=138)$.

\begin{tabular}{|c|c|c|c|c|c|c|c|}
\hline & \multirow{2}{*}{ Hypothesis } & \multirow{2}{*}{ Mediator(s) } & \multirow{2}{*}{ Indirect effect } & \multirow{2}{*}{$B$} & \multirow{2}{*}{ SE } & \multicolumn{2}{|c|}{$95 \% \mathrm{BCa} \mathrm{CI}$} \\
\hline & & & & & & Lower & Upper \\
\hline \multirow{3}{*}{ Specific effects } & 1 & $i m p_{\text {self }}$ & $a_{1} b_{1}$ & 0.089 & 0.080 & -0.038 & 0.287 \\
\hline & 2 & $i m p_{\text {proxy }}$ & $a_{2} b_{2}$ & 0.073 & 0.114 & -0.121 & 0.340 \\
\hline & 3 & Threat perception & $a_{3} b_{3}$ & 0.244 & 0.162 & 0.019 & 0.703 \\
\hline \multirow{4}{*}{ Serial effects } & 4 & $i m p_{\text {self }}-i m p_{\text {proxy }}$ & $a_{1} d_{21} b_{2}$ & 0.016 & 0.028 & -0.022 & 0.097 \\
\hline & 5 & imp $p_{\text {self }}$ - threat perception & $a_{1} d_{31} b_{3}$ & 0.010 & 0.026 & -0.017 & 0.112 \\
\hline & 6 & $i m p_{\text {proxy }}$ - threat perception & $a_{1} d_{32} b_{3}$ & 0.048 & 0.050 & -0.001 & 0.210 \\
\hline & 7 & $i m p_{\text {self }}-i m p_{\text {proxy }}-$ threat perception & $a_{1} d_{21} d_{32} b_{3}$ & 0.010 & 0.014 & 0.000 & 0.066 \\
\hline
\end{tabular}

Note. imp $_{\text {self }}=$ impairment based on self-report; $\mathrm{imp}_{\text {proxy }}=$ impairment based on parent report.

child's pain after treatment [41]. Some studies have examined threat perceptions in children as well. In an earlier study, Lipani and Walker [42] identified that child's threat appraisal is associated with maternal distress and impairment in family functioning. Walker and colleagues [22] reported a high correspondence between child- and parent-reported threat perceptions. Assuming that threat perceptions are significant beliefs for various outcomes in childhood pain and in order to gain a deeper understanding on family processes in chronic pain [28], future studies should explore correlates of threat perception on the family level as well. By including a broad age range as well as both self- and proxy reports of impairment, we aimed to clarify the relative influence of impairment on HCU. We hypothesized that, for pediatric health care seeking, parental perceptions play a major role, even for adolescents, who may be more autonomous in terms of medical visits. $27.2 \%$ of our sample was aged 13-17 years. Increasing child age was accompanied by increasing values on all variables except for self-reported impairment. Our results suggest that the parental perspective is the more influential source of information, probably acting through their perception of threat, in explaining HCU.

The results of this study have to be seen in the context of methodological strengths and limitations. According to recommendations for the assessment of pain [30, 43], our model assessed pain in child self-report. We used a retrospective cross-sectional recall by the children to cover the last 2 weeks. While a retrospective recall might be biased, a recent study in children and adolescents aged 8-18 years suffering irritable bowel syndrome did not find significant differences between retroversus prospective diary approaches for pain assessment [44]. Nonetheless, our retrospective approach of pain assessment has to be seen as limitation. In line with current reviews and recommendations on the interplay of child and parent factors $[28,45]$, we integrated both childand parent-report of impairment into one mediation analysis. Furthermore, by analyzing impairment and parental threat perception concurrently in one analysis, we were able to analyze their distinct contribution for HCU. The sample covered a broad age range, and a possible confounding age effect was statistically controlled for. With regard to methodological aspects, the serial model implies causal links between the mediators. However, the cross-sectional design precludes causal conclusions, as the interrelations between variables in the model might be epiphenomenal, due to one common cause, or even causal in nature [37]. Future research should longitudinally analyze causal agents for parental threat perception and HCU. While the application of an OLS-regression-based analysis and underlying $t$-distribution ensures more accurate $p$ values for the path coefficients in smaller samples like in this study [37], research with larger sample sizes would enable path analysis configurations and estimations of latent variable models.

The generalizability of the results might be limited due to the preselected clinical sample in secondary care and the fact that the sample was well-educated, with over $80 \%$ of parents having a medium to high educational background. In addition, a larger sample size across various diagnostic subgroups might allow for intergroup differences in the meditational model and distinct pathways. In this study, separate analyses were not conducted as the model lacked statistical power. Reliance on parent report may lead to shared method variance accounting for relations among threat perception and HCU; however, by analyzing pain and impairment based on child report, we aimed to reduce this limitation.

Our findings allow several implications for future research and clinical practice. Based on our results, parents' threat perception might constitute one approach to health care utilization, underlining the central role parents play in frequent pediatric visits. According to parents' individual threat evaluation, doctor visits may be adequate reactions to a child's chronic abdominal pain. However, the potential course from an adaptive behavior to highly increased medical utilization as maladaptive coping needs to be studied in future research. Longitudinal analysis of outcomes in high-utilizing families is warranted. Threat perception seems to only partly reflect child's pain and related impairment, as these variables explained $24.7 \%$ of variance of threat perception in our sample. In clinical practice, a thorough examination of what actually drives threat perception should be undertaken. Not only are increased psychological symptoms highly prevalent among parents of children suffering from CAP [46, 47], maternal psychological well-being was identified as distinct characteristic in consulting families as well [13]. Therefore, 
one further can assume that parental symptoms of anxiety or health anxiety might influence their threat perception concerning child's abdominal pain and also pediatric HCU as well. This relationship needs to be studied in future research.

Our results suggest that addressing parental threat perception might be a fruitful component in parent-centered counseling in the pediatric setting. Accompanied by skilled health care communication [48], delivering information about the natural course and prognosis of abdominal pain can specifically target the threat perception. This might help to foster a biopsychosocial understanding of illness and enable effective coping and adjustment for both child and parent. It has been shown that a biopsychosocial conceptual model of CAP positively influences the course of CAP [49] and might reduce excessive HCU as well [9]. Our results stress that we should not only aim to modify behavioral responses to the child's pain complaints, but should also target parents' painspecific appraisals.

\section{Conclusions}

As chronic abdominal pain is the most common reason for consultations of pediatric gastroenterologists, this study aimed to identify significant pathways to pain-related medical seeking in secondary care. When parental threat perception is taken into account, the degree of pain-related impairment in functioning did not significantly contribute to health care seeking, neither in child, nor in parent report. Only parent's threat perception significantly linked child's pain to the number of medical visits. Therefore, the parental influence is mainly acting through their subjective perception of threat, less through their perception of child's impairment. Targeting parental threat perception in the pediatric setting might be a promising way to foster a biopsychosocial model of chronic pain and that way to reduce increased health care use.

\section{Competing Interests}

The authors declare no conflict of interests.

\section{Acknowledgments}

The authors wish to thank the pediatric gastroenterologists for their cooperation and recruitment of patients: Dr. Rüdiger Adam (Mannheim), Dr. Antje Ballauff (Krefeld), Dr. Sebastian Becker (Darmstadt), Dr. Michael Friedt (Düsseldorf), Dr. Ulrich Gabel (Oberursel), Dr. Ute Kloß (Berlin), Dr. Benno Kretschmar (Eisenach), Dr. Silke Petau (Berlin), Dr. Carsten Posovszky (Ulm), Dr. Raymund Pothmann (Hamburg), Professor Doctor Michael Radke (Potsdam), Dr. Markus Richter (Augsburg), Dr. Maike Schier (Hamburg), Dr. Oliver Schirrmacher (Vechta), and Dr. Markus Schmitt (Ludwigsfelde). They also thank Dr. Martina Groß for recruitment and especially all participating children, parents, and clinic staff members for their engagement.

\section{References}

[1] U. Ellert, H. Neuhauser, and A. Roth-Isigkeit, "Pain in children and adolescents in Germany: the prevalence and usage of medical services. Results of the German Health Interview and Examination Survey for Children and Adolescents (KiGGS)," Bundesgesundheitsblatt Gesundheitsforschung Gesundheitsschutz, vol. 50, no. 5, pp. 711-715, 2007.

[2] M. Vila, T. Kramer, J. E. Obiols, and M. E. Garralda, "Abdominal pain in British young people: associations, impairment and health care use," Journal of Psychosomatic Research, vol. 73, no. 6, pp. 437-442, 2012.

[3] A. Roth-Isigkeit, U. Thyen, H. Stöven, J. Schwarzenberger, and P. Schmucker, "Pain among children and adolescents: restrictions in daily living and triggering factors," Pediatrics, vol. 115, no. 2, pp. e152-e162, 2005.

[4] P. Warschburger, J. Hänig, M. Friedt, C. Posovszky, M. Schier, and C. Calvano, "Health-related quality of life in children with abdominal pain due to functional or organic gastrointestinal disorders," Journal of Pediatric Psychology, vol. 39, no. 1, pp. 45$54,2014$.

[5] T. A. Coffelt, B. D. Bauer, and A. E. Carroll, "Inpatient characteristics of the child admitted with chronic pain," Pediatrics, vol. 132, no. 2, pp. e422-e429, 2013.

[6] I. J. D. Schwille, K. E. Giel, U. Ellert, S. Zipfel, and P. Enck, "A community-based survey of abdominal pain prevalence, characteristics, and health care use among children," Clinical Gastroenterology and Hepatology, vol. 7, no. 10, pp. 1062-1068, 2009.

[7] G. Dhroove, A. Chogle, and M. Saps, "A million-dollar workup for abdominal pain: is it worth it?" Journal of Pediatric Gastroenterology and Nutrition, vol. 51, no. 5, pp. 579-583, 2010.

[8] M. M. Lane, E. M. Weidler, D. I. Czyzewski, and R. J. Shulman, "Pain symptoms and stooling patterns do not drive diagnostic costs for children with functional abdominal pain and irritable bowel syndrome in primary or tertiary care," Pediatrics, vol. 123, no. 3, pp. 758-764, 2009.

[9] K. J. Lindley, D. Glaser, and P. J. Milla, "Consumerism in healthcare can be detrimental to child health: lessons from children with functional abdominal pain," Archives of Disease in Childhood, vol. 90, no. 4, pp. 335-337, 2005.

[10] J. Miró, A. Huguet, and R. Nieto, "Predictive factors of chronic pediatric pain and disability: a delphi poll," The Journal of Pain, vol. 8, no. 10, pp. 774-792, 2007.

[11] J. V. Campo, D. M. Comer, L. Jansen-McWilliams, W. Gardner, and K. J. Kelleher, "Recurrent pain, emotional distress, and health service use in childhood," Journal of Pediatrics, vol. 141, no. 1, pp. 76-83, 2002.

[12] D. M. Janicke, J. W. Finney, and A. W. Riley, "Children's health care use: a prospective investigation of factors related to careseeking," Medical Care, vol. 39, no. 9, pp. 990-1001, 2001.

[13] R. L. Levy, S. L. Langer, L. S. Walker, L. D. Feld, and W. E. Whitehead, "Relationship between the decision to take a child to the clinic for abdominal pain and maternal psychological distress," Archives of Pediatrics and Adolescent Medicine, vol. 160, no. 9, pp. 961-965, 2006.

[14] C. W. Perquin, J. A. M. Hunfeld, A. A. J. M. HazebroekKampschreur et al., "Insights in the use of health care services in chronic benign pain in childhood and adolescence," Pain, vol. 94, no. 2, pp. 205-213, 2001.

[15] M. Toliver-Sokol, C. B. Murray, A. C. Wilson, A. Lewandowski, and T. M. Palermo, "Patterns and predictors of health service utilization in adolescents with pain: comparison between a 
community and a clinical pain sample," The Journal of Pain, vol. 12, no. 7, pp. 747-755, 2011.

[16] N. K. Venepalli, M. A. L. Van Tilburg, and W. E. Whitehead, "Recurrent abdominal pain: what determines medical consulting behavior?" Digestive Diseases and Sciences, vol. 51, no. 1, pp. 192-201, 2006.

[17] F. M. Blyth, L. M. March, A. J. M. Brnabic, and M. J. Cousins, "Chronic pain and frequent use of health care," Pain, vol. 111, no. 1-2, pp. 51-58, 2004.

[18] J. V. Campo, L. Jansen-Mcwilliams, and D. M. Comer, "Somatization in pediatric primary care: association with psychopathology, functional impairment, and use of services," Journal of the American Academy of Child and Adolescent Psychiatry, vol. 38, no. 9, pp. 1093-1101, 1999.

[19] K. E. Boerner, M. Noel, K. A. Birnie, L. Caes, M. Petter, and C. T. Chambers, "Impact of threat level, task instruction, and individual characteristics on cold pressor pain and fear among children and their parents," Pain Practice, vol. 16, no. 6, pp. 657668, 2016.

[20] L. Caes, T. Vervoort, Z. Trost, and L. Goubert, "Impact of parental catastrophizing and contextual threat on parents' emotional and behavioral responses to their child's pain," Pain, vol. 153, no. 3, pp. 687-695, 2012.

[21] T. Vervoort, L. Caes, Z. Trost, L. Notebaert, and L. Goubert, "Parental attention to their child's pain is modulated by threatvalue of pain," Health Psychology, vol. 31, no. 5, pp. 623-631, 2012.

[22] L. S. Walker, A. L. Sherman, S. Bruehl, J. Garber, and C. A. Smith, "Functional abdominal pain patient subtypes in childhood predict functional gastrointestinal disorders with chronic pain and psychiatric comorbidities in adolescence and adulthood," Pain, vol. 153, no. 9, pp. 1798-1806, 2012.

[23] R. L. Levy, S. L. Langer, L. S. Walker et al., "Cognitive-behavioral therapy for children with functional abdominal pain and their parents decreases pain and other symptoms," American Journal of Gastroenterology, vol. 105, no. 4, pp. 946-956, 2010.

[24] R. L. Levy, S. L. Langer, L. S. Walker et al., "Twelve-month follow-up of cognitive behavioral therapy for children with functional abdominal pain," JAMA Pediatrics, vol. 167, no. 2, pp. 178-184, 2013.

[25] V. L. Champion and C. S. Skinner, "The health belief model," in Health Behavior and Health Education: Theory, Research, and Practice, K. Glanz, B. K. Rimer, and K. Viswanath, Eds., pp. 4565, Jossey-Bass, San Francisco, Calif, USA, 4th edition, 2008.

[26] P. Lavielle and N. Wacher, "The predictors of glucose screening: the contribution of risk perception," BMC Family Practice, vol. 15, article 108, 2014.

[27] M. Connelly, K. K. Anthony, and L. E. Schanberg, "Parent perceptions of child vulnerability are associated with functioning and health care use in children with chronic pain," Journal of Pain and Symptom Management, vol. 43, no. 5, pp. 953-960, 2012.

[28] T. M. Palermo, C. R. Valrie, and C. W. Karlson, "Family and parent influences on pediatric chronic pain," American Psychologist, vol. 69, no. 2, pp. 142-152, 2014.

[29] D. L. Wong and C. M. Baker, "Pain in children: comparison of assessment scales," Pediatric Nursing, vol. 14, no. 1, pp. 9-17, 1988.

[30] D. Tomlinson, C. L. von Baeyer, J. N. Stinson, and L. Sung, "A systematic review of faces scales for the self-report of pain intensity in children," Pediatrics, vol. 126, no. 5, pp. el168-e1198, 2010.
[31] L. S. Walker and J. W. Greene, "Children with recurrent abdominal pain and their parents: more somatic complaints, anxiety, and depression than other patient families?" Journal of Pediatric Psychology, vol. 14, no. 2, pp. 231-243, 1989.

[32] C. Hermann, J. Hohmeister, K. Zohsel, M.-L. Tuttas, and H. Flor, "The impact of chronic pain in children and adolescents: development and initial validation of a child and parent version of the Pain Experience Questionnaire," Pain, vol. 135, no. 3, pp. 251-261, 2008.

[33] B. Hübner, T. Hechler, M. Dobe et al., "Pain-related disability in adolescents suffering from chronic pain: preliminary examination of the Pediatric Pain Disability Index (P-PDI)," Schmerz, vol. 23, no. 1, pp. 20-32, 2009.

[34] N. D. Weinstein, "Perceived probability, perceived severity, and health-protective behavior," Health Psychology, vol. 19, no. 1, pp. 65-74, 2000.

[35] J. Van Der Pligt, "Perceived risk and vulnerability as predictors of precautionary behaviour," British Journal of Health Psychology, vol. 3, no. 1, pp. 1-14, 1998.

[36] P. H. Ditto, J. B. Jemmott III, and J. M. Darley, "Appraising the threat of illness: a mental representational approach," Health Psychology, vol. 7, no. 2, pp. 183-201, 1988.

[37] A. F. Hayes, Introduction to mediation, moderation and conditional process analysis. A regression-based approach, The Guilford Press, New York, NY, USA, 2013.

[38] K. J. Preacher and A. F. Hayes, "SPSS and SAS procedures for estimating indirect effects in simple mediation models," Behavior Research Methods, Instruments, \& Computers, vol. 36, no. 4, pp. 717-731, 2004.

[39] J. L. Schafer and J. W. Graham, "Missing data: our view of the state of the art," Psychological Methods, vol. 7, no. 2, pp. 147-177, 2002.

[40] K. J. Preacher and A. F. Hayes, "Asymptotic and resampling strategies for assessing and comparing indirect effects in multiple mediator models," Behavior Research Methods, vol. 40, no. 3, pp. 879-891, 2008.

[41] R. L. Levy, S. L. Langer, J. M. Romano et al., "Cognitive mediators of treatment outcomes in pediatric functional abdominal pain," Clinical Journal of Pain, vol. 30, no. 12, pp. 1033-1043, 2014.

[42] T. A. Lipani and L. S. Walker, "Children's appraisal and coping with pain: relation to maternal ratings of worry and restriction in family activities," Journal of Pediatric Psychology, vol. 31, no. 7, pp. 667-673, 2006.

[43] L. L. Cohen, K. Lemanek, R. L. Blount et al., "Evidence-based assessment of pediatric pain," Journal of Pediatric Psychology, vol. 33, no. 9, pp. 939-955, 2008.

[44] M. M. Self, A. E. Williams, D. I. Czyzewski, E. M. Weidler, and R. J. Shulman, "Agreement between prospective diary data and retrospective questionnaire report of abdominal pain and stooling symptoms in children with irritable bowel syndrome," Neurogastroenterology \& Motility, vol. 27, no. 8, pp. 1110-1119, 2015.

[45] S. Kaartina, Y. S. Chin, R. Fara Wahida et al., "Adolescent selfreport and parent proxy-report of health-related quality of life: an analysis of validity and reliability of PedsQL ${ }^{\mathrm{TM}} 4.0$ among a sample of Malaysian adolescents and their parents," Health and Quality of Life Outcomes, vol. 13, no. 1, article 44, 2015.

[46] J. V. Campo, J. Bridge, A. Lucas et al., "Physical and emotional health of mothers of youth with functional abdominal pain," Archives of Pediatrics and Adolescent Medicine, vol. 161, no. 2, pp. 131-137, 2007. 
[47] P. G. Ramchandani, L. Murray, G. Romano, H. Vlachos, and A. Stein, "An investigation of health anxiety in families where children have recurrent abdominal pain," Journal of Pediatric Psychology, vol. 36, no. 4, pp. 409-419, 2011.

[48] M. Levetown, "Communicating with children and families: from everyday interactions to skill in conveying distressing information," Pediatrics, vol. 121, no. 5, pp. el441-e1460, 2008.

[49] E. Crushell, M. Rowland, M. Doherty et al., "Importance of parental conceptual model of illness in severe recurrent abdominal pain," Pediatrics, vol. 112, no. 6, part 1, pp. 1368-1372, 2003. 


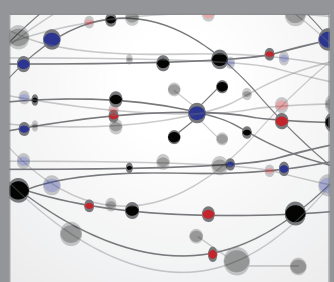

The Scientific World Journal
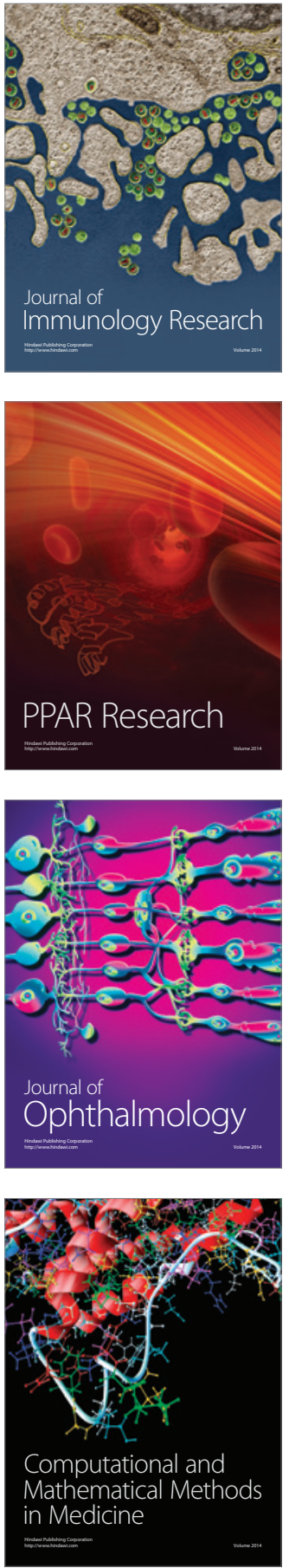

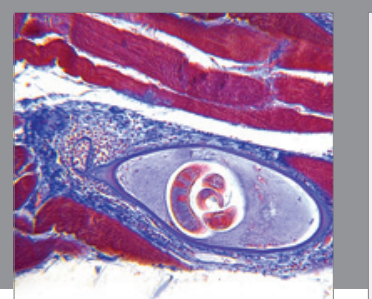

Gastroenterology Research and Practice

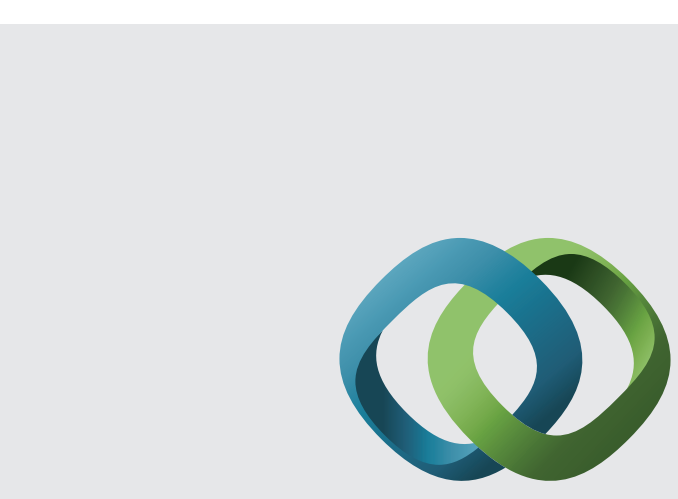

\section{Hindawi}

Submit your manuscripts at

http://www.hindawi.com
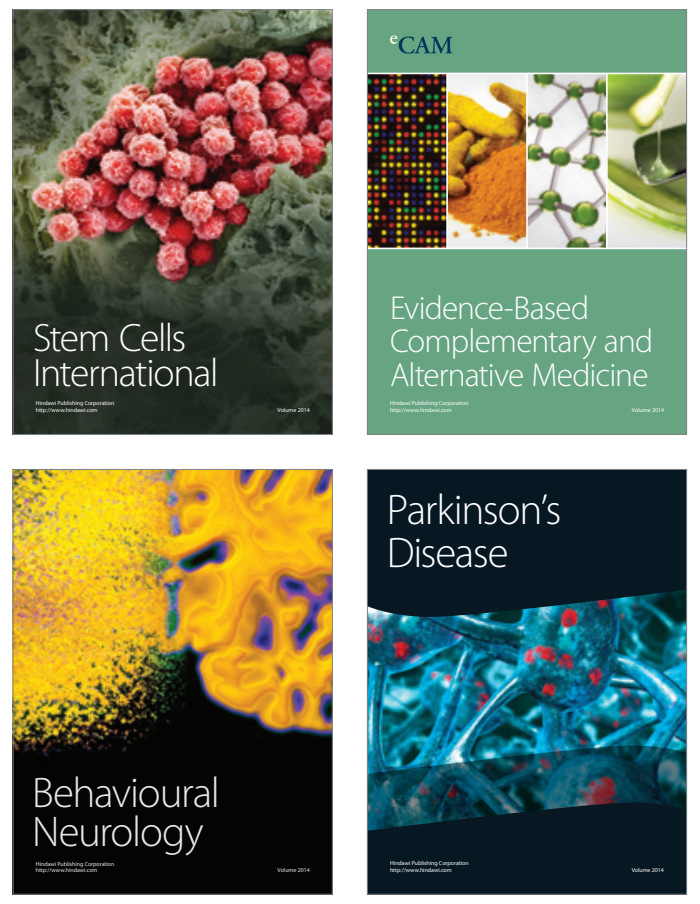
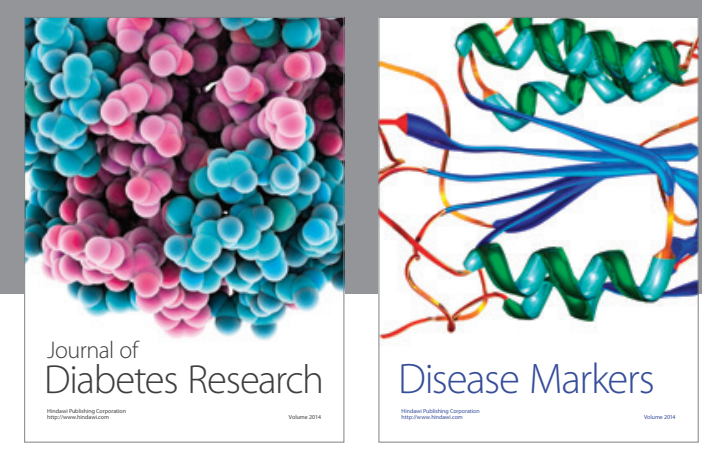

Disease Markers
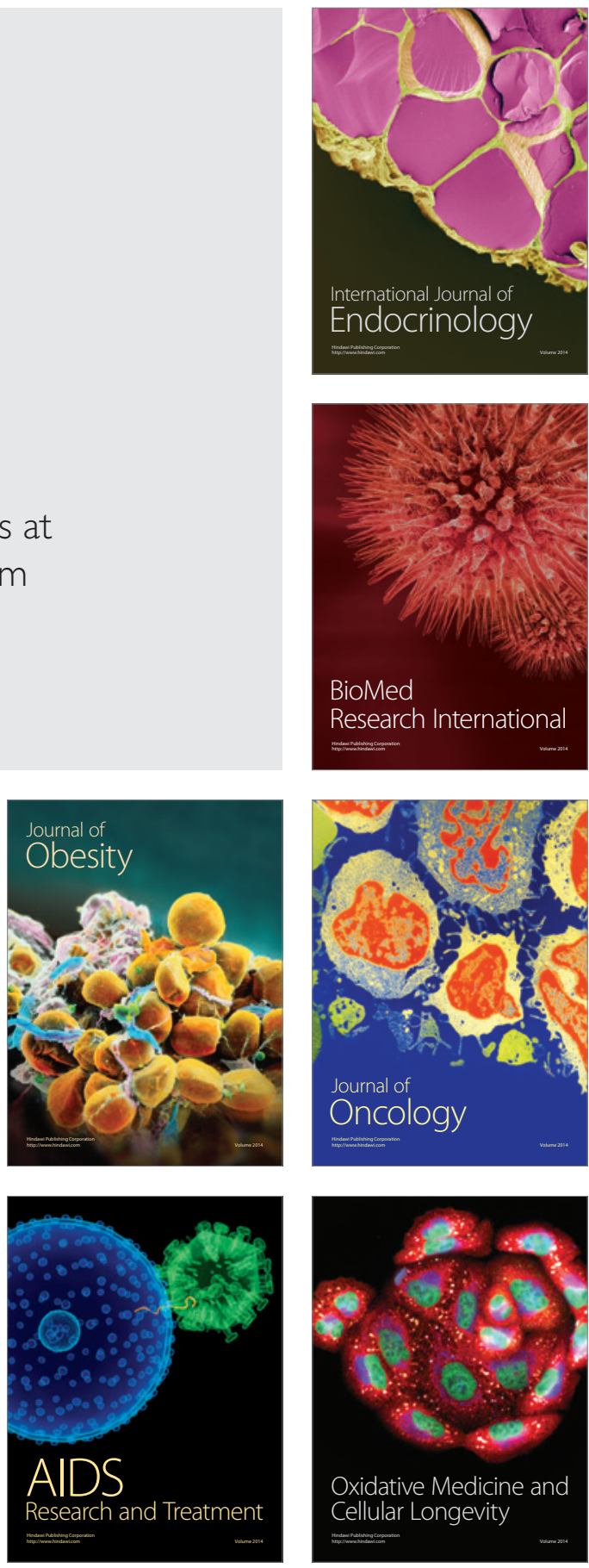